\title{
Approximate Axial Symmetries from Continuous Time Quantum Walks
}

\author{
Luca Rossi ${ }^{1}$, Andrea Torsello ${ }^{1}$, and Edwin R. Hancock ${ }^{2}$ \\ 1 Department of Environmental Science, Informatics and Statistics, \\ Ca' Foscari University of Venice, Italy \\ 2 Department of Computer science, University of York, UK
}

\begin{abstract}
The analysis of complex networks is usually based on key properties such as small-worldness and vertex degree distribution. The presence of symmetric motifs on the other hand has been related to redundancy and thus robustness of the networks. In this paper we propose a method for detecting approximate axial symmetries in networks. For each pair of nodes, we define a continuous-time quantum walk which is evolved through time. By measuring the probability that the quantum walker to visits each node of the network in this time frame, we are able to determine whether the two vertices are symmetrical with respect to any axis of the graph. Moreover, we show that we are able to successfully detect approximate axial symmetries too. We show the efficacy of our approach by analysing both synthetic and real-world data.
\end{abstract}

Keywords: Complex Network, Symmetry, Quantum Walk.

\section{Introduction}

The study of complex networks 1 has recently attracted considerable interest because of the large variety of complex systems that can be modeled and analysed using graphs. A non-exhaustive list of examples includes metabolic networks [2, protein interactions [3], brain networks [4, vascular systems [5], scientific collaboration networks [6] and road maps [7]. Properties such as small-worldness and the power-law distribution of vertex degrees [1] have been observed in several real-world networks, suggesting a marked difference with Erdös-Rényi random graphs [8].

More recently there has been some interest in characterizing the presence of symmetries in networks 9 [10. Recall that, given a graph $G=(V, E)$, an automorphism is a permutation $\sigma$ of the set of vertices $V$ of the graph which preserve the adjacency relations, i.e. if $(u, v) \in E$ then $(\sigma(u), \sigma(v)) \in E$. Hence we can view the group of automorphisms $\operatorname{Aut}(G)$ of a graph as a representation of its symmetries. MacArthur et al. 9] observe that many real-world graphs possess a very large automorphism group, in contrast to classical random graph models. In particular the authors observe the presence of a certain number of small symmetric subgraphs, such as tree-like or clique-like structures, and relate this to the redundancy and thus robustness of real-world networks. Note however 
that the problem of finding the set of automorphisms of a graph is actually an instance of the graph isomorphism problem, and thus it belongs to the NP class. Xiao et al. 10] study the origin of symmetry in real-world networks. In common with [9], their work is based on the analysis of local symmetric motifs such as symmetric bicliques, i.e. an induced complete bipartite subgraph, denoted as $K_{V_{1}, V_{2}}$, in which every vertex of $V_{1}$ is connected to every vertex of $V_{2}$. Their analysis reveals that the symmetry of complex networks is a consequence of a particular linkage pattern, where vertices with similar degrees tend to share common neighbors. It is also worth mentioning the work of Mowshowitz [11, which links the complexity of a graph to the entropy of the distribution of symmetric orbits.

Recently there has been a considerable interest in quantum walks, as an alternative to the well studied classical random walks. Although similar in its definition, the quantum walk is remarkably different from its classical counterpart. Most notably, its evolution is governed by a unitary matrix instead of a stochastic one and the state vector is complex valued instead of real valued. This in turn produces interference effects which yield completely different probability distributions on the graph. Moreover, these interference effects seem to be enhanced by the presence of symmetrical motifs in the graph. Emms et al. 12 showed that quantum commute time embeddings are tightly related to the presence of symmetries. In particular the authors found that the embedding co-ordinates of nodes are degenerate in dimensions that correspond to global symmetries. In a related paper, Emms et al. 13 demonstrate how to lift the cospectrality of strongly regular graphs using the third power of the support matrix derived from a discrete time quantum walk. Thus it seems reasonable to investigate the use of quantum walks as a means of detecting symmetries in networks.

In real-world data, however, we have to deal with the presence of noise, which will eventually break the symmetries of the network. In this paper we propose a new method for detecting the approximate axial symmetries of a graph using continuous time quantum walks. The remainder of this paper is organised as follows. First we review the definition of the continuous time quantum walk on a graph, then we show how to exploit the interference patterns to detect both exact and approximate axial symmetries and then we briefly discuss the proposed algorithm. Finally our approach is evaluated on a set of synthetic graphs and real-world networks.

\section{Continuous-Time Quantum Walks}

Quantum walks are the quantum analogue of classical random walks [14. In this paper we consider only continuous-time quantum walks, as first introduced by Farhi and Gutmann in [15].

As in the classical random walk, given a graph $G=(V, E)$, the state space of the continuous-time quantum walk defined on $G$ is the set of the vertices $V$ of the graph. Unlike the classical case, where the evolution of the walk is governed by a stochastic matrix (i.e. a matrix whose columns sum to unity), in 
the quantum case the dynamics of the walker is governed by a complex unitary matrix i.e., a matrix that multiplied by its conjugate transpose yields the identity matrix. Hence the evolution of the quantum walk is reversible, which implies that quantum walks are non-ergodic and do not possess a limiting distribution. Using Dirac notation, we denote the basis state corresponding to the walk being at vertex $u \in V$ as $|u\rangle$. A general state of the walk is a complex linear combination of the basis states, such that the state of the walk at time $t$ is defined as

$$
\left|\psi_{t}\right\rangle=\sum_{u \in V} \alpha_{u}(t)|u\rangle
$$

where the amplitude $\alpha_{u}(t) \in \mathbb{C}$ and $\left|\psi_{t}\right\rangle \in \mathbb{C}^{|V|}$ are both complex.

At each point in time the probability of the walker being at a particular vertex of the graph is given by the square of the norm of the amplitude of the relative state. More formally, let $X^{t}$ be a random variable giving the location of the walker at time $t$. Then the probability of the walker being at the vertex $u$ at time $t$ is given by

$$
\operatorname{Pr}\left(X^{t}=u\right)=\alpha_{u}(t) \alpha_{u}^{*}(t)
$$

where $\alpha_{u}^{*}(t)$ is the complex conjugate of $\alpha_{u}(t)$. Moreover $\sum_{u \in V} \alpha_{u}(t) \alpha_{u}^{*}(t)=1$ and $\alpha_{u}(t) \alpha_{u}^{*}(t) \in[0,1]$, for all $u \in V, t \in \mathbb{R}^{+}$.

We now introduce the evolution operator of the quantum walk. First though, recall that the adjacency matrix of the graph $G$ has elements

$$
A_{u v}=\left\{\begin{array}{l}
1 \text { if }(u, v) \in E \\
0 \text { otherwise }
\end{array}\right.
$$

Let $D$ be the diagonal degree matrix with elements $d_{u}=\sum_{v=1}^{n} A(u, v)$, where $n=|V|$ is the number of vertices of the graph. The Laplacian of $G$ is then defined as the degree matrix minus the adjacency matrix, i.e. $L=D-A$.

The evolution of the walk is then given by Schrödinger equation, where we take the Hamiltonian of the system to be the graph Laplacian, which yields

$$
\frac{d}{d t}\left|\psi_{t}\right\rangle=-i L\left|\psi_{t}\right\rangle
$$

Given an initial state $\left|\psi_{0}\right\rangle$, we can solve Equation廿 4 to determine the state vector at time $t$

$$
\left|\psi_{t}\right\rangle=e^{-i L t}\left|\psi_{0}\right\rangle
$$

Given the Laplacian matrix we can compute its spectral decomposition $L=$ $\Phi \Lambda \Phi^{T}$, where $\Phi$ is the $n \times n$ matrix $\Phi=\left(\phi_{1}\left|\phi_{2}\right| \ldots \mid \phi_{n}\right)$ with the ordered eigenvectors as columns and $\Lambda=\operatorname{diag}\left(\lambda_{1}, \lambda_{2}, \ldots, \lambda_{n}\right)$ is the $n \times n$ diagonal matrix with the ordered eigenvalues as elements, such that $0=\lambda_{1} \leq \lambda_{2} \leq \ldots \leq \lambda_{n}$.

Using the spectral decomposition of the graph Laplacian and the fact that $\exp [-i L t]=\Phi^{\top} \exp [-i \Lambda t] \Phi$ we can finally write

$$
\left|\psi_{t}\right\rangle=\Phi^{\top} e^{-i \Lambda t} \Phi\left|\psi_{0}\right\rangle
$$




\section{Approximate Axial Symmetries Detection}

In order to detect the axial symmetries of a graph, we exploit the interference properties exhibited by quantum walks. In particular, our analysis will rely on the destructive interference which arises when a symmetrical structure is present. Note, however, that we are interested in both exact and approximate axial symmetries. In fact, due to the presence of different noise sources, most real-world networks are not perfectly symmetric. In consequence, the search for exact axial symmetries would fail to discover those global symmetries which are more likely to be affected by noise. On the other hand, we argue that our algorithm is capable of detecting both exact and approximate axial symmetries, thus making it suitable for real-world network analysis.

\subsection{Methodology}

Given a pair of vertices $u, v$, we initialise the quantum walk as follows

$$
\alpha_{j}(0)=\left\{\begin{array}{l}
+\frac{1}{\sqrt{2}} \text { if } j=u \\
-\frac{1}{\sqrt{2}} \text { if } j=v \\
0 \text { otherwise }
\end{array}\right.
$$

If $u$ and $v$ are symmetrical with respect to a symmetry axis $A$, then it is easy to show that when the walk is initialised as above we have $\alpha_{w}(t)=0, \forall w \in A$ and $\forall t$.

Theorem 1. If $u, v$ are symmetrical with respect to a symmetry axis $A$ and $\alpha_{u}(0)=-\alpha_{v}(0)$, then $\alpha_{w}(t)=0, \forall w \in A$ and $\forall t$.

Sketch of Proof. Assume that the graph $G$ has at least one symmetry axis $A$, and $w \in A$ is a vertex of $G$. Then, for each path from $u$ to $w$, there will be a symmetrical path from $v$ to $w$. As a consequence of this, both walkers starting from $u$ and from $v$ will arrive at $w$ at the same time $t$. Moreover, since we initialised the amplitudes such that $\alpha_{u}(0)=-\alpha_{v}(0)$, the two walkers will be in antiphase and thus their contribution to the observation probability of $w$ will cancel out, i.e. $\operatorname{Pr}\left(X^{t}=w\right)=0, \forall t$.

Note that due to its oscillatory behaviour, the observation probability of the quantum walker on any node might temporarily collapse to zero. However, only if the vertex belongs to a symmetry axis its observation probability will remain constantly null.

Hence the procedure to detect the symmetry axes in a graph is as follows. First we define a quantum walk according to Equation 7 . We then let the quantum walk evolve for a time interval $T$ and we measure the total observation probability $\pi_{w}=\sum_{t \in T} \operatorname{Pr}\left(X^{t}=w\right)$ for each node $w \neq u, v$ during $T$. If $\pi_{w}=0$, then we say that the node belongs to the symmetry axis. We repeat this procedure for each pair of nodes of the graph, and we detect all the exact symmetry axes of the network. Finally, we can estimate the symmetry axes sizes by counting, for a given pair of nodes, the number of nodes $w$ where $\pi_{w}=0$. 


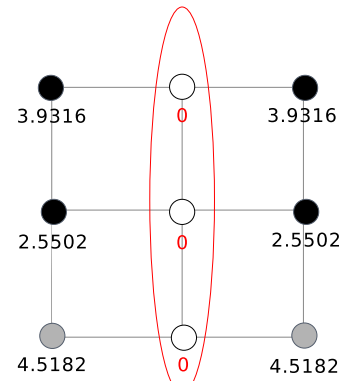

(a) $3 \times 3$ grid

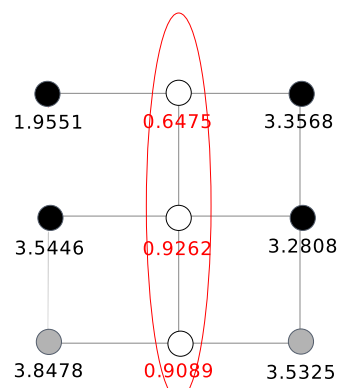

(b) noisy $3 \times 3$ grid

Fig. 1. When applied to a $3 \times 3$ grid, our algorithm is able to detect all its 4 symmetry axes. Moreover, even if some noise is present, a suitable choice of $\tau$ will still allow us to detect the original axes

Figure 1(a) shows the result of running the proposed algorithm on a $3 \times 3$ grid. If we initialise the bottom corners of the graph with equal but opposite amplitudes, we see that the observation probabilities of the vertices belonging to the vertical axis remain constantly equal to zero. In particular here we let the quantum walk evolve from $t=0$ to $t=10$, sampling this time interval 100 times uniformly. For each axial node $w$ we have $\pi_{w}=0$, while the nodes with initial non-zero amplitudes have the highest total observation probability. The remainder of the vertices of the graph have $\pi_{w}>0$, and symmetrically placed nodes with respect to the vertical axis share the same $\pi_{w}$. Note that we detect the same axis if we choose another pair of vertices which are symmetrical with respect to this axis, such as the top corners.

Moreover, we argue that we can detect approximate axial symmetries as well, i.e., axial symmetries which are affected by noise, by selecting those vertices in which $\pi<\tau$, where $\tau$ is a suitably chosen threshold. Figure 1(b) shows what happens if we add some noise the the $3 \times 3$ grid by removing one edge. Note that due to the small size of the graph the deletion of one single edge can actually deeply change the graph structure. Although the total observation probability has clearly changed and it is now non-zero everywhere, we still see that it is lower on the vertices corresponding to the vertical axis. Hence, by choosing a suitable value for $\tau$, we are still able to detect this approximate symmetry.

\section{Experimental Results}

In this section, we validate the proposed approach by performing a series of experiments on both synthetic data and real-world data.

\subsection{Synthetic Data}

The synthetic data is composed of Erdös-Rényi random graphs [8], small-world graphs, scale-free graphs, stochastic Kronecker graphs [16] (which exhibit both 


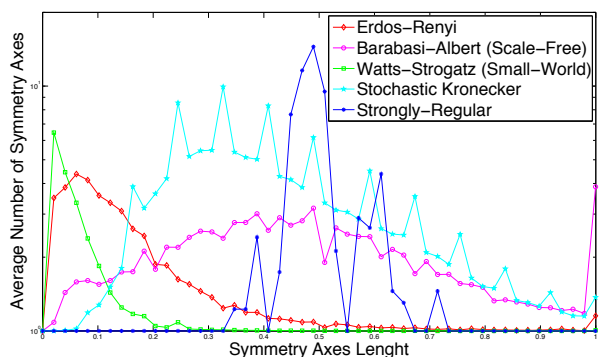

(a) $\tau=0.2$

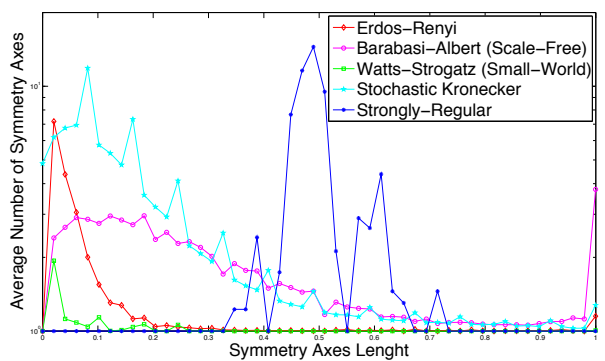

(c) $\tau=0.1$

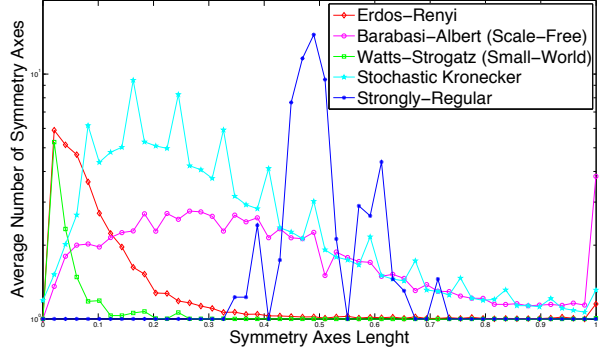

(b) $\tau=0.15$

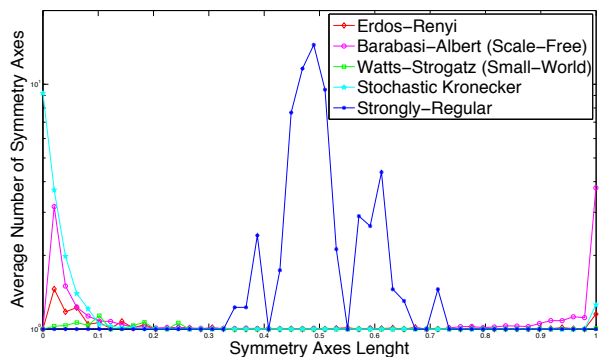

(d) $\tau=0.01$

Fig. 2. Symmetry axes distribution. Note that as the threshold varies, the shape of the strongly-regular graphs distribution remains unaltered, as the symmetry present in this category are all exact.

small-world and scale-free properties), and strongly regular graphs. A regular graph with $\nu$ vertices and degree $k$ is said to be strongly regular if there are two integers $\lambda$ and $\mu$ such that every two adjacent vertices have $\lambda$ common neighbours and every two non-adjacent vertices have $\mu$ common neighbors. We choose strongly regular graphs because they are known to be highly symmetric and this should be reflected in the experimental results.

For each graph in the dataset, we compute its symmetry axes together with their sizes, as explained in the previous section. Figure 2 shows the distribution of the symmetry axes length for each type of network, for different choices of the threshold $\tau$. Note that local symmetries correspond to larger axes, since the axis size is equal to the number of nodes of the graph minus the size of the symmetric orbit, which in the case of a local symmetry is clearly small. On the other hand, a global symmetry will correspond to smaller symmetry axis. In other words, a left peaked distribution indicates the presence of global symmetries, while a right peaked distribution indicates the presence of local symmetries.

Note that the distribution for the strongly-regular graphs remains unaltered when we change $\tau$. This is because the graphs in this category possess exact symmetries, due to their regular structure. Hence the probability of the walker being found at a node belonging to a symmetry axis is exactly zero and we recover the same axes independently of the threshold value. Note, moreover, 

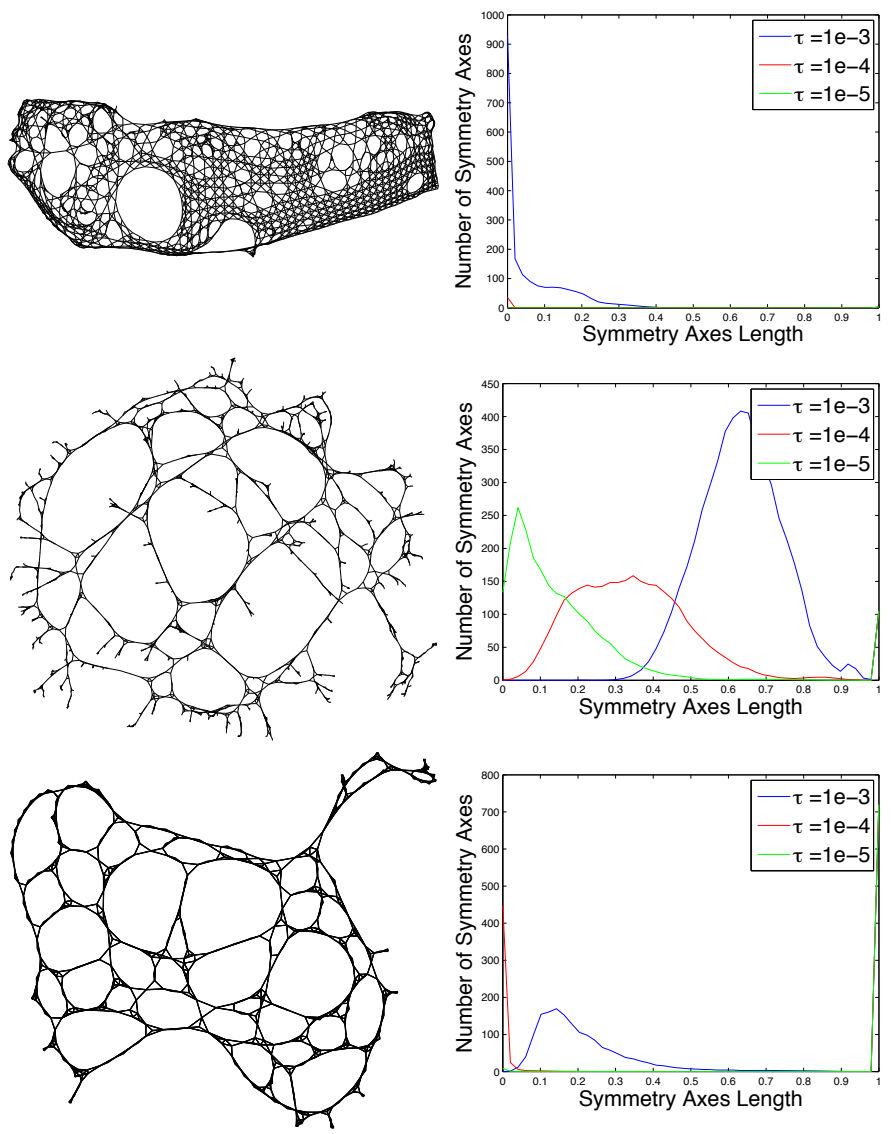

Fig. 3. Sample cities embeddings and their corresponding axes length distributions. Note how different layouts give rise to different symmetries.

that the high number of symmetry axes belonging to this class of graphs is exactly what we would expect given the high degree of symmetry displayed by strongly-regular graphs.

As for the other network models, Figure 2 shows that the number of exact symmetries is clearly lower. We observe the presence of a high number of exact local symmetries in the scale-free graphs, which are probably due to the presence of small trees rooted in a hub node. It is interesting to note that the behaviour of the stochastic Kronecker graphs, which possess both scale-free and small-world properties, seems to be dominated by their scale-free behaviour, although the number of local symmetries is clearly reduced. More generally, Figure 2 shows that we can easily separate graphs belonging to different network models on the basis of their symmetry axes distributions. 

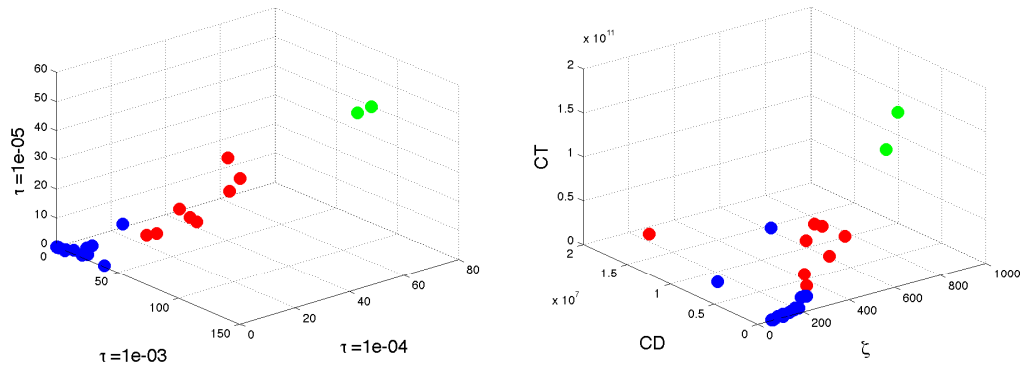

Fig. 4. City maps embeddings obtained using the mean of the symmetry axes plot distributions for three different values of $\tau$ as co-ordinates (left). The cities are coloured according to the labels induced by k-means. The labeling is consistent when the embedding is done using Communicability Distance 1], the classical Commute Time and the zeta function as co-ordinates (right), but there are still some differences which suggest that the information extracted with our algorithm is meaningful and novel.

\subsection{Real-World Data}

Road networks are a typical example of technological networks, i.e. man-made networks designed for the distribution of resources. Other examples include power grids, airline routes, river networks and the Internet. In this paper we apply our algorithm to a dataset of 33 city maps. For each city, we compute the approximate symmetry axes and their length. Figure 3 shows the embeddings of 3 different cities and the corresponding plots. We observe that different layouts of the cities give rise to different symmetries. As expected, the first city, which shows a very regular grid-like structure, seems to possess only approximate global symmetries, but no perfect symmetries. On the other hand, the second city displays a wide variety of approximate symmetry axes, and a few exact local symmetries, similarly to those displayed by the scale-free network model. A visual inspection of its embedding confirms the presence of several small hubs, as predicted. Finally, the third city shows a large number of local symmetries, which arise as a consequence of its very particular linkage pattern, where pairs of nodes are connected in an quasi-bipartite fashion.

In order to take the analysis on step further, we describe each city with a threedimensional feature vector whose co-ordinates are respectively the means of the axes length distributions for decreasing thresholds. The resulting embedding is shown in Figure 4 (left). Here we can clearly see 3 well separated clusters, where the labels of the clusters have been assigned using k-means. Note that each city of Figure 3 actually belongs to a different cluster of Figure 4 . Moreover, we compare our embedding with the one obtained using the Communicability Distance [1, the classical Commute Time and the zeta function $\zeta(s)=\sum_{\lambda_{i} \neq 0} \lambda_{i}^{-s}$ associated with the Laplacian eigenvalues as co-ordinates. Figure 4 (right) shows that the result is still quite consistent with our labeling, although we can clearly see some differences. This indicates that the our algorithm is indeed extracting some meaningful and novel information from the data. 


\section{Conclusions}

In this paper we proposed a method for detecting approximate axial symmetries in networks. For each pair of nodes of the graph, we define a continuous-time quantum walk which is evolved through time. By measuring the probability of the quantum walker to visit each node of the network in this time frame, we are able to tell whether if two vertices are symmetrical with respect to any axis of the graph. Moreover, we showed that we are able to successfully detect approximate axial symmetries as well. We demonstrated the efficacy of our approach by analysing both synthetic and real-world data.

Acknowledgments. Edwin Hancock was supported by a Royal Society Wolfson Research Merit Award.

\section{References}

1. Estrada, E.: The Structure of Complex Networks. Oxford University Press (2011)

2. Jeong, H., Tombor, B., Albert, R., Oltvai, Z., Barabási, A.: The large-scale organization of metabolic networks. Nature 407, 651-654 (2000)

3. Ito, T., Chiba, T., Ozawa, R., Yoshida, M., Hattori, M., Sakaki, Y.: A comprehensive two-hybrid analysis to explore the yeast protein interactome. Proceedings of the National Academy of Sciences 98, 4569 (2001)

4. Sporns, O.: Network analysis, complexity, and brain function. Complexity 8, 56-60 (2002)

5. West, G., Brown, J., Enquist, B.: A general model for the structure, function, and allometry of plant vascular systems. Nature 400, 664-667 (1999)

6. Newman, M.: Scientific collaboration networks. i. network construction and fundamental results. Physical Review E 64, 016131 (2001)

7. Kalapala, V., Sanwalani, V., Moore, C.: The structure of the united states road network. University of New Mexico (2003) (preprint)

8. Erdös, P., Rényi, A.: On random graphs. Publ. Math. Debrecen 6, 290-297 (1959)

9. MacArthur, B., Sánchez-García, R., Anderson, J.: Symmetry in complex networks. Discrete Applied Mathematics 156, 3525-3531 (2008)

10. Xiao, Y., Xiong, M., Wang, W., Wang, H.: Emergence of symmetry in complex networks. Phys. Rev. E 77, 066108 (2008)

11. Mowshowitz, A.: Entropy and the complexity of graphs: I. an index of the relative complexity of a graph. Bulletin of Mathematical Biology 30, 175-204 (1968)

12. Emms, D., Wilson, R., Hancock, E.: Graph Embedding Using Quantum Commute Times. In: Escolano, F., Vento, M. (eds.) GbRPR. LNCS, vol. 4538, pp. 371-382. Springer, Heidelberg (2007)

13. Emms, D., Severini, S., Wilson, R., Hancock, E.: Coined Quantum Walks Lift the Cospectrality of Graphs and Trees. In: Rangarajan, A., Vemuri, B.C., Yuille, A.L. (eds.) EMMCVPR 2005. LNCS, vol. 3757, pp. 332-345. Springer, Heidelberg (2005)

14. Kempe, J.: Quantum random walks: an introductory overview. Contemporary Physics 44, 307-327 (2003)

15. Farhi, E., Gutmann, S.: Quantum computation and decision trees. Physical Review A 58, 915 (1998)

16. Mahdian, M., Xu, Y.: Stochastic Kronecker Graphs. In: Bonato, A., Chung, F.R.K. (eds.) WAW 2007. LNCS, vol. 4863, pp. 179-186. Springer, Heidelberg (2007) 